\title{
LUSUI: DESENVOLVIMENTO DE LISTA DE VERIFICAÇÃO DE USABILIDADE PARA INTERFACES DE SMARTPHONE COM FOCO NO USUÁRIO IDOSO
}

\section{LUSUI: DEVELOPMENT OF A USABILITY CHECKLIST FOR SMARTPHONE INTERFACES FOCUSED ON ELDER PEOPLE}

\author{
Marcos Souza Filho ${ }^{1}$, M.Sc. \\ contato@marcostenorio.com https://orcid.org/0000-0002-5905-739X \\ Fábio Campos ${ }^{1}, \mathrm{PhD}$ \\ fc2005@gmail.com https://orcid.org/0000-0002-7882-876X
}

\author{
${ }^{1}$ Programa de Pós Graduação em Design, UFPE, Recife, PE, Brasil
}

Usabilidade, Smartphone, Idosos, Envelhecimento.

O presente artigo descreve os procedimentos de pesquisa e criação de uma lista de verificação de usabilidade focada na interface de smartphones, com o intuito de propor melhorias na experiência do público idoso. Foi realizada pesquisa bibliográfica com o intuito de identificar quais principais dificuldades encontradas pelos usuários, além de identificar as soluções já existentes e desenvolvidas anteriormente. Após a identificação das possíveis soluções, foi proposta a criação de uma lista de verificação para projetos de interfaces direcionado a esse público, validada por um grupo de especialistas, que se propõe a auxiliar desenvolvedores na tarefa de identificar possíveis lacunas a serem preenchidas durante o projeto.

\section{Usability, Smartphone, Elder, Aging}

This article describes the research procedures and creation of a usability checklist focused on the smartphone interface, to propose improvements in the experience of the elderly audience. A bibliographical research was carried out in order to know the main difficulties encountered by users, in addition to identifying existing and previously developed solutions. After identifying the possible solutions, it was brought to create a checklist for interface projects aimed at this audience, validated by a group of experts, which proposes to assist developers in the task of identifying potential gaps to be filled during the project. 


\section{Introdução}

Durante a pandemia do novo corona vírus, decretada pela Organização Mundial da Saúde no final de 2019, idosos foram inseridos na cultura digital e expostos a vários problemas como maior risco de morte com a contaminação pelo vírus, isolamento social e solidão trazidas pelas restrições sociais e a dificuldade no acesso à informação e serviços de qualidade trazida pela inserção tardia na cultura digital (BO XIE et al, 2020).

Nos estudos divulgados antes da pandemia (OPAS, 2018), as estimativas de crescimento da população de idosos era de 2 bilhões de pessoas até 2050 , tornando-se $25 \%$ da população mundial e, para efeito comparativo, o Brasil seguiria essa tendência, tornando-se o quinto lugar em número de idosos no mundo, com uma população que em 2030 já seria maior que o número de crianças e jovens de até 14 anos. Por conta dos impactos na saúde pública, causados pelo corona vírus, ainda não se sabe como ficará essa projeção.

A tecnologia está cada vez mais acessível a todos os públicos e os idosos, que sempre foram tão avessos aos artefatos digitais, estão mais conectados e abertos a experimentar as facilidades trazidas pelos smartphones. Segundo a pesquisa PNAD Contínua TIC 2017 (IBGE, 2018), o número de pessoas com acesso à internet aumentou, em um ano, em 10,2 milhões de usuários, entre esses, 2,3 milhões são de idosos. Elevando a porcentagem de idosos conectados de $24,7 \%$ para 31,1\% no período de 2016 para 2017.

O crescimento foi significativo e mostra o quão importante é a necessidade de possibilitar melhores condições de usabilidade e acesso desse público aos artefatos. Além disso, o mesmo estudo relata que 97\% das pessoas que acessam a internet no país, o fazem através de smartphones, contrapondo o número de pessoas que acessam através de computadores, que caiu de $63 \%$ para $56,6 \% \mathrm{em}$ um ano. Ademais, dentre os idosos, o Instituto (IBGE, 2018) declara que $63,5 \%$ das pessoas com mais de 60 anos possuem acesso a um celular ou smartphone.

O cenário brasileiro reflete a realidade mundial, em um estudo do Pew Research Center (PEW RESEARCH CENTER, 2017), cerca de 74\% dos idosos que participaram da pesquisa, declaram não se sentirem totalmente confortáveis em utilizar os smartphones. As queixas relatadas vão desde os desafios físicos na operação do artefato até a familiaridade com a linguagem e funcionalidade da tecnologia. O que leva ao número de $73 \%$ deles que precisam de auxílio para utilizar seus aparelhos. Ainda assim, mais de $58 \%$ acreditam que a tecnologia pode trazer impactos positivos para a sociedade.

Recentemente, devido ao isolamento social, foi possível observar que esse acesso às novas tecnologias foi mais incentivado pela pandemia do novo corona vírus, em contraponto com o número de empresas que se interessam em desenvolver soluções para os idosos que é estimado em apenas $30 \%$ (CNN, 2021).

Observando o número cada vez mais expressivo de idosos conectados através de smartphones questiona-se:

- as ferramentas de apoio ao projeto de interfaces com foco em usabilidade atendem às questões específicas do público idoso?

Partindo desse questionamento, com a revisão bibliográfica, a pesquisa buscou identificar heurísticas e listas de verificação de usabilidade existentes que se preocupam com a questão dos idosos no uso de smartphones, foram feitas buscas em repositórios digitais e livros com o intuito de listar as recomendações já publicadas e as lacunas existentes nelas.

Ao longo do tempo, diversos autores abordam questões de usabilidade gerais em interfaces desde a década de 1990 e mais recentemente, com a popularização da informática e do smartphone, as mais específicas do publico idoso vêm à tona. Independente da especificidade, recomendações de acessibilidade podem contribuir para a experiência de uso de um artefato por diversos públicos, o que as tornam parte da abordagem desse estudo. 
Isto posto, esse artigo irá relatar os procedimentos adotados na averiguação e no desenvolvimento de uma proposta de lista de verificação de usabilidade de smartphones, com base na literatura existente, visando questões específicas do público idoso em busca da melhoria da interface na etapa projetual dos aplicativos e sistemas operacionais.

\section{Idosos e suas necessidades específicas com a tecnologia}

Um estudo do Pew Research Center (PEW RESEARCH CENTER, 2019) sobre o comportamento de usuários de celulares e smartphones nos Estados Unidos, a quantidade de idosos que utilizam os aparelhos móveis são de $91 \%$, desses apenas 39\% utilizam celulares mais simples que smartphones. Desses, 12\% consideram-se dependentes de smartphones para suas atividades diárias. Por mais que esse número possa parecer tímido, o estudo vem acompanhando o crescimento da utilização a cada ano e em 2013, essa realidade era de $3 \%$, em sete anos um aumento expressivo de $400 \%$.

Por mais que ainda se sintam com alguma dificuldade para utilizar os smartphones, a intenção deles é de utilizar a ferramenta para algumas atividades que irão facilitar o seu cotidiano, o site especializado Aging in Place, listou algumas dessas atividades mais comuns entre eles (AGING IN PLACE, 2021):

1- $\quad$ Fazer telefonemas (principalmente de emergência);

2- Vídeo chamadas para os netos;

3- $\quad$ Controle de dispositivos inteligentes;

4- $\quad$ Mensagens de texto para amigos;

5- $\quad$ GPS para caminhas e/ou dirigir;

6- Verificar as horas e data;

7- $\quad$ Monitoramento de saúde por meio de aplicativos dedicados;

8- $\quad$ Acesso a internet.

O site ainda lista algumas das facilidades que podem ser oferecidas ao público como Entretenimento, maior capacidade de compartilhamento, praticidade por meio de aplicativos, estímulos - principalmente às atividades físicas-, utilização de assistentes virtuais, entre outras atividades. Em contraponto, lista também as desvantagens do aparelho como: frustração no uso, excesso de opções, custos altos, possibilidade de roubos, gastos acidentais - como compras acidentais em aplicativos - e dificuldades para configuração. (AGING IN PLACE, 2021)

Assim como os outros públicos, os idosos têm interesse nas novas tecnologias e nas facilidades trazidas por elas, mas enfrentam barreiras que frustram sua experiência e que poderiam ser resolvidas com estudos e projetos mais preocupados com a usabilidade mais direcionada para eles.

\section{Métodos de inspeção de usabilidade: Heurísticas e Listas de Verificação}

A usabilidade é definida por diversos autores e normas, mas podemos resumir em dois conceitos mais proeminentes como a ISO 9241-210 (ISO, 2010) que a define como o quanto um produto pode ser usado por usuários específicos, para atingir metas específicas, com eficácia, eficiência e satisfação em um contexto de uso específico. No Brasil, a principal definição é estabelecida pelo Inmetro a partir da norma NBR 9241-11 (ABNT, 2011), que reafirma o conceito internacional.

A inspeção de usabilidade pode ser medida em ambientes controlados, envolvendo usuários, em ambientes naturais, também com os usuários ou em qualquer ambiente, sem a presença dos usuários, como as listas de 
verificação e as avaliações heurísticas (PREECE, ROGERS, \& SHARP, 2019). Dentre as variadas técnicas, as heurísticas destacam-se por apresentar vantagens como a facilidade de aplicação, o custo reduzido, a possibilidade de executá-la desde o início do desenvolvimento do projeto até as etapas finais, além de serem aplicáveis para qualquer tamanho de sistemas, dos mais complexos aos mais simples. (CIBYS, BETIOL, \& FAUST, 2017)

\subsection{Heurísticas de Usabilidade}

As heurísticas de usabilidade foram criadas por Nielsen e Molich (1990) e complementadas por Nielsen posteriormente (NIELSEN, 1994), para serem utilizadas como recomendações para designers e desenvolvedores durante as etapas de desenvolvimento de interfaces para sistemas computacionais. As heurísticas consideram questões de uso e cognitivas e fazem recomendações de melhorias de acordo com dez princípios que tratam de questões relativas a: Visibilidade do estado do sistema; Similaridade entre o sistema e o mundo real; Controle e liberdade do usuário; Consistência e padrões; Prevenção de erros; reconhecer ao invés de memorizar; Eficiência e flexibilidade de uso; Estética e design minimalista; ajude o usuário a reconhecer, diagnosticar e recuperar-se de erros. e Ajuda e documentação.

Essas heurísticas passaram por diversas revisões durante o tempo e foram sendo adaptadas para contextos específicos, desde as novas plataformas como smartphones e tablets até para que atendessem necessidades específicas de públicos que, com o tempo, percebeu-se não serem atendidos pelas primeiras heurísticas. Algumas dessas atualizações que podem ser citadas dentre as inúmeras heurísticas são: Princípios de Norman - PN (NORMAN, 2018), Critérios Ergonômicos de Usabilidade - CE (BASTIEN \& SCAPIN, 1993), Regras de Ouro - RO (SHNEIDERMAN \& PLAISANT, 2004); Além das específicas para dispositivos móveis: Heurísticas de avaliação móvel - HG (GÓMEZ, CABALLERO, \& SEVILLANO, 2014), Usabilidade Móvel - NB (NIELSEN \& BUDIU, Usabilidade Móvel, 2014), Heurísticas para avaliação de usabilidade de launchers de smartphones para idosos - AL (AL-RAZGAN, AL-KHALIFA, \& AL-SHAHRANI, 2014), Heurísticas de avaliação de usabilidade de aplicativos fitness em smartphones para idosos - SHN (SILVA, HOLDEN, \& NII, 2014) e as Recomendações de usabilidade para interface visando o público idoso - AG (ANJOS \& GONTIJO, 2015).

Utilizando as heurísticas listadas anteriormente, foi desenvolvido um quadro comparativo entre as similaridades encontradas entre elas para resumir suas principais características de forma mais simplificada. Utilizando a proposta desenvolvida por Al-Razgan et al (2014), foram agrupadas as dificuldades em quatro categorias: Facilidade de Leitura, Interação, Memória e Funcionalidades, essa escolha se deveu a ser o estudo mais específico sobre a usabilidade de smartphones para o público idoso. Na primeira coluna são elencadas as dificuldades encontradas pelo público e nas colunas seguintes, marcadas com um ' $X$ ', quando presentes ou sem marcação quando ausentes, estão as heurísticas citadas:

\begin{tabular}{|c|c|c|c|c|c|c|c|c|c|c|}
\hline \multicolumn{2}{|l|}{ Dificuldade } & HN & PN & RO & CE & HG & NB & $A L$ & SHN & AG \\
\hline \multirow{10}{*}{$\begin{array}{c}\text { Facilidade de } \\
\text { Leitura }\end{array}$} & D1. Alto Contraste & & & & & & & $\mathbf{x}$ & $\mathbf{x}$ & $\mathbf{x}$ \\
\hline & D2. Acessibilidade & & & & $\mathbf{x}$ & & & & $\mathbf{x}$ & $\mathbf{x}$ \\
\hline & D3. Tamanho dos botões na tela & & & & & & $\mathbf{x}$ & $\mathbf{x}$ & $x$ & $\mathbf{x}$ \\
\hline & D4. Ícones relacionados à realidade & $\mathbf{x}$ & & & $\mathbf{x}$ & $\mathbf{x}$ & $\mathbf{x}$ & $\mathbf{x}$ & $\mathbf{x}$ & $\mathbf{x}$ \\
\hline & D5. Tamanho do texto na tela & & & & & & & $\mathbf{x}$ & $x$ & $\mathbf{x}$ \\
\hline & $\begin{array}{l}\text { D6. Evitar o uso de termos técnicos } \\
\text { (ícone, atalho, link, contatos) }\end{array}$ & $\mathbf{x}$ & $\mathbf{x}$ & & & $\mathbf{x}$ & & $\mathbf{x}$ & & $\mathbf{x}$ \\
\hline & D7. Limitar o número de itens na tela & $\mathbf{x}$ & & & $\mathbf{x}$ & $\mathbf{x}$ & $\mathbf{x}$ & $\mathbf{x}$ & $\mathbf{x}$ & \\
\hline & $\begin{array}{l}\text { D8. Utilização de metáforas visuais } \\
\text { coerentes }\end{array}$ & $\mathbf{x}$ & $\mathbf{x}$ & & $\mathbf{x}$ & $x$ & & $x$ & $\mathbf{x}$ & $\mathbf{x}$ \\
\hline & D9. Sistema se adapta ao usuário & $\mathbf{x}$ & & & $\mathbf{x}$ & $\mathbf{x}$ & & & & \\
\hline & D10. Possibilitar leitor de tela & & & & & & & & & \\
\hline
\end{tabular}




\begin{tabular}{|c|c|c|c|c|c|c|c|c|c|c|}
\hline & D11. Evitar categorização por cores & & & & & & & & $\mathbf{x}$ & $\mathbf{x}$ \\
\hline & $\begin{array}{l}\text { D12. Facilitar Alteração de configurações } \\
\text { de Acessibilidade }\end{array}$ & & & & & & & & $\mathbf{x}$ & $\mathbf{x}$ \\
\hline & D13. Espaçamento entre itens & & & & & & & & $x$ & $x$ \\
\hline & $\begin{array}{l}\text { D14. Evitar Animações e movimentos } \\
\text { rápidos }\end{array}$ & & & & & & & & $\mathbf{x}$ & $\mathbf{x}$ \\
\hline \multirow{14}{*}{ Interação } & D15. Sistema é agradável para o usuário & & & & & $\mathbf{x}$ & & & & \\
\hline & D16. Maior sensibilidade ao toque & & & & & & & & & \\
\hline & D17. Feedback das ações & $\mathbf{x}$ & $\mathbf{x}$ & $\mathbf{x}$ & $\mathbf{x}$ & $\mathbf{x}$ & & $\mathbf{x}$ & $\mathbf{x}$ & $\mathbf{x}$ \\
\hline & D18. Gestual simplificado & & $\mathbf{x}$ & & & & & $\mathbf{x}$ & & \\
\hline & $\begin{array}{l}\text { D19. Oferece a escrita simplificada com } \\
\text { palavras disponíveis em botões maiores }\end{array}$ & & & & & & & & & \\
\hline & $\begin{array}{l}\text { D20. Fornece possibilidade de } \\
\text { compartilhar assim que fotografa }\end{array}$ & & & & & & & & & \\
\hline & D21. Sons simples e claros & & & & & & & $\mathbf{x}$ & $\mathbf{x}$ & \\
\hline & $\begin{array}{l}\text { D22. O sistema apresenta suas opções } \\
\text { de forma clara }\end{array}$ & $\mathbf{x}$ & $\mathbf{x}$ & & $\mathbf{x}$ & $\mathbf{x}$ & & $\mathbf{x}$ & $\mathbf{x}$ & $\mathbf{x}$ \\
\hline & D23. Consistência dos elementos visuais & $\mathbf{x}$ & $\mathbf{x}$ & $\mathbf{x}$ & $\mathbf{x}$ & $\mathbf{x}$ & & $\mathbf{x}$ & $\mathbf{x}$ & $\mathbf{x}$ \\
\hline & $\begin{array}{l}\text { D24. Itens disponibilizados em locais que } \\
\text { facilitam a interação }\end{array}$ & & $\mathbf{x}$ & & $\mathbf{x}$ & & & $\mathbf{x}$ & $\mathbf{x}$ & $x$ \\
\hline & $\begin{array}{l}\text { D25. Sinalização de localização na } \\
\text { interface }\end{array}$ & $\mathbf{x}$ & $\mathbf{x}$ & $\mathbf{x}$ & $\mathbf{x}$ & $\mathbf{x}$ & $\mathbf{x}$ & $\mathbf{x}$ & & $\mathbf{x}$ \\
\hline & D26. Feedback Tátil, Visual e Sonoro & & & & & & & & $x$ & $x$ \\
\hline & D27. Evitar Menus suspensos & & & & & & & & $\mathbf{x}$ & \\
\hline & D28. Evitar rolagem de tela & & & & & & & & & \\
\hline \multirow{8}{*}{ Memória } & D29. Reconhecer ao invés de lembrar & $\mathbf{x}$ & $\mathbf{x}$ & $\mathbf{x}$ & & $\mathbf{x}$ & & & $\mathbf{x}$ & \\
\hline & D30. Memória da atividade & $\mathbf{x}$ & $\mathbf{x}$ & & & $\mathbf{x}$ & $\mathbf{x}$ & & & \\
\hline & $\begin{array}{l}\text { D31. Possibilidade de voltar ao ponto } \\
\text { anterior }\end{array}$ & $\mathbf{x}$ & $\mathbf{x}$ & $\mathbf{x}$ & & $\mathbf{x}$ & $x$ & & $\mathbf{x}$ & $\mathbf{x}$ \\
\hline & D32. Possibilidade de desfazer ações & $\mathbf{x}$ & & $\mathbf{x}$ & & $\mathbf{x}$ & & & & \\
\hline & $\begin{array}{l}\text { D33. Agrupamento de funções } \\
\text { semelhantes }\end{array}$ & & & $\mathbf{x}$ & $\mathbf{x}$ & & & $\mathbf{x}$ & & \\
\hline & $\begin{array}{l}\text { D34. Disponibilidade de funções básicas } \\
\text { (telefone, mensagem, desligar, câmera, } \\
\text { etc.) }\end{array}$ & & & & & & & $\mathbf{x}$ & & $\mathbf{x}$ \\
\hline & $\begin{array}{l}\text { D35. Considera o conhecimento do } \\
\text { usuário }\end{array}$ & & $\mathbf{x}$ & & & $\mathbf{x}$ & & & $\mathbf{x}$ & $\mathbf{x}$ \\
\hline & D36. Limitar a uma tarefa por vez & & & & & & & & $\mathbf{x}$ & \\
\hline \multirow{9}{*}{ Funcionalidades } & D37. Reconhecer comandos de voz & & & & & & & & & \\
\hline & D38. Atalho para emergências & & & & & & & & & \\
\hline & D39. Prevenção de Erros & $\mathbf{x}$ & $\mathbf{x}$ & $\mathbf{x}$ & $\mathbf{x}$ & $\mathbf{x}$ & & $\mathbf{x}$ & $\mathbf{x}$ & $\mathbf{x}$ \\
\hline & $\begin{array}{l}\text { D40. Fornecer informações do estado do } \\
\text { sistema }\end{array}$ & $\mathbf{x}$ & $\mathbf{x}$ & $\mathbf{x}$ & $\mathbf{x}$ & $\mathbf{x}$ & & $\mathbf{x}$ & $\mathbf{x}$ & $\mathbf{x}$ \\
\hline & $\begin{array}{l}\text { D41. Fornecer ajuda para qualquer tela } \\
\text { do sistema }\end{array}$ & $\mathbf{x}$ & & & & $\mathbf{x}$ & & & $\mathbf{x}$ & $\mathbf{x}$ \\
\hline & $\begin{array}{l}\text { D42. Fornecer documentação auxiliar de } \\
\text { ajuda }\end{array}$ & $\mathbf{x}$ & & & & $\mathbf{x}$ & & & $\mathbf{x}$ & \\
\hline & $\begin{array}{l}\text { D43. Informar corretamente como } \\
\text { resolver erros }\end{array}$ & $\mathbf{x}$ & $\mathbf{x}$ & $\mathbf{x}$ & $\mathbf{x}$ & $\mathbf{x}$ & & & & \\
\hline & $\begin{array}{l}\text { D44. Proporcionar sensação de controle } \\
\text { ao usuário }\end{array}$ & & $\mathbf{x}$ & $\mathbf{x}$ & $\mathbf{x}$ & & & & $\mathbf{x}$ & $\mathbf{x}$ \\
\hline & D45. Privacidade & & & & & $\mathbf{x}$ & & & & \\
\hline
\end{tabular}

Tabela 01: Quadro comparativo de heurísticas de usabilidade Fonte: Os autores, 2019

Esse estudo foi além da coleta de heurísticas existentes, ele identifica de que forma elas podem ser aplicadas de um modo a incentivar a melhoria da usabilidade dos smartphones pensados para idoso, assim, como produto derivado das heurísticas, serão estudadas as listas de verificação de usabilidade 


\subsection{Listas de Verificação de Usabilidade}

As Heurísticas são aplicáveis em contextos específicos, compostos por um grupo de especialistas em usabilidade, não é recomendável que sejam utilizadas por profissionais de outras áreas e isso pode, em algum momento, dificultar a sua aplicação. Uma forma de facilitar ainda mais a inspeção de usabilidade é a criação de listas de verificação de usabilidade, que podem ser baseadas em observações de contextos de uso baseado na tarefa ou até mesmo nas próprias heurísticas.

Conhecidas também como checklists, permitem a identificação de problemas menores de usabilidade, sem a necessidade de aplicação por meio de especialistas em usabilidade (CYBIS, et al., 2003). Complementando a utilidade das listas de verificação e sua importância, Brykczynski (1999) explica que elas podem ser utilizadas para avaliar questões relativas à interface, ao código, aos requisitos do sistema durante os testes, os documentos de ajuda e utilização de processos executados durante a utilização do sistema avaliado.

Cybis et al (2003) ainda defendem as listas de verificação como técnica capaz de identificar problemas gerais e repetitivos da interface e, como vantagens principais, apresenta: rapidez na aplicação, sistematização da aplicação por profissionais especializados, independentemente de serem especialistas em usabilidade, pois o conhecimento ergonômico já está inserido na própria ferramenta. Além disso, em outra publicação, Cybis et al (2017) ainda explicam que as listas servem de base para inspeção de conformidades com as normas e reforçam que a forma como elas conduzem a avaliação é um ponto positivo de sua utilização, além de sistemática e objetiva, torna a pesquisa mais econômica.

Corroborando o exposto, Matias, conclui que as mais significantes vantagens da utilização de listas de verificação são: Possibilidade de realização por projetistas não especialistas em IHC , design ou usabilidade; facilidade de identificar problemas gerais e repetitivos; custo reduzido; sistematização que garante estabilidade nos resultados encontrados; redução na subjetividade da avaliação, pois o avaliador é direcionado para pontos específicos da interface; maior aperfeiçoamento da interface avaliada com a correção dos problemas identificados pela lista de verificação. Todavia, vale salientar que o mesmo autor ainda afirma que nada pode substituir a pesquisa com usuários reais, que pode gerar dados muito mais embasados.

A utilização das listas de verificação pode produzir resultados mais uniformes e abrangentes, além de mais objetivos, mas Cybis (2017) e Hom (2003) recomendam a aplicação das listas concomitantemente aos testes de usabilidade com usuários ou avaliações heurísticas, pois elas podem direcionar a inspeção para pontos específicos, que podem ser confirmados com a lista de verificação.

É possível encontrar algumas listas de verificação de forma digital ou analógica, desde a própria ISSO 9241 até as disponíveis em sites como o SAPO, de Portugal. Algumas das listas figuram entre as mais conhecidas no Brasil, como: ERGOLIST (LabIUtil, 2011), TICESE (GAMEZ, 1998), ACESSIBILIDADE (LabIUtil, 2002), ERGOCOIN, CTI - MEDE-PROS, MATcH (GQS-UFSC, n.d.) e a SAPO. Para concentrar as características resumidas e cada uma das listas de verificação analisadas nesse estudo, foi elaborada uma tabela com base nos estudos de Dyck (2005), que reuniu as principais listas de verificação com o intuito de identificar a estrutura padrão das listas e desenvolveu um quadro comparativo, adaptado pelos autores para inserção de uma nova lista - SAPO - e explicitado a seguir: 
Ergodesign \& HCI

número 1, volume 9, jan-jun (2021)
PUC-Rio Pontifícia Universidade Católica do Rio de Janeiro Departamento de Artes \& Design I PPGDesign

LEUI I Laboratório de Ergodesign e Usabilidade de Interfaces

\begin{tabular}{|c|c|c|c|c|c|c|c|c|}
\hline & 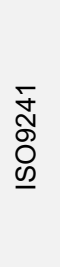 & 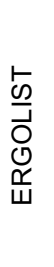 & $\begin{array}{l}\underset{\tilde{S}}{\tilde{U}} \\
\stackrel{\underline{U}}{F}\end{array}$ & 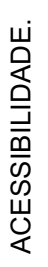 & $\begin{array}{l}\text { z } \\
\text { Oे } \\
\text { O } \\
\text { O } \\
\stackrel{y}{\Psi}\end{array}$ & 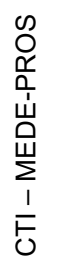 & $\frac{\frac{T}{0}}{\frac{1}{6}}$ & $\begin{array}{l}\text { O } \\
\text { ळ } \\
\text { 心 }\end{array}$ \\
\hline \multicolumn{9}{|l|}{ Objetivos do checklist } \\
\hline Avaliação Somativa (nota final) & & & $x$ & & & $x$ & $\mathrm{X}$ & \\
\hline Avaliação Formativa (revisões de projeto) & $\mathrm{X}$ & $\mathrm{X}$ & & $\mathrm{X}$ & $\mathrm{X}$ & & $\mathrm{X}$ & $\mathbf{x}$ \\
\hline \multicolumn{9}{|l|}{ Organização das questões } \\
\hline Qualidades & & $\mathrm{X}$ & $x$ & $\mathrm{X}$ & $\mathrm{X}$ & & $\mathrm{X}$ & $\mathbf{X}$ \\
\hline Componentes & $x$ & & & & $x$ & & & \\
\hline \multicolumn{9}{|l|}{ Apresentação das questões } \\
\hline Título & $\mathrm{x}$ & $\mathrm{x}$ & & & & & $\mathrm{X}$ & $\mathbf{x}$ \\
\hline Enunciado textual & $\mathrm{x}$ & $\mathrm{x}$ & $x$ & $\mathrm{X}$ & $\mathrm{X}$ & & $\mathrm{X}$ & $\mathbf{x}$ \\
\hline Exemplo & $\mathrm{x}$ & $\mathrm{x}$ & & $\mathrm{X}$ & & & $\mathrm{X}$ & $\mathbf{x}$ \\
\hline Notas esclarecedoras & $x$ & $x$ & & $\mathrm{X}$ & & & & $\mathbf{x}$ \\
\hline Glossário & $x$ & $\mathrm{X}$ & & $\mathrm{X}$ & & & & \\
\hline Justificativa da importância do quesito & & $\mathrm{x}$ & & $\mathrm{X}$ & & & & $\mathbf{x}$ \\
\hline Tipo de transformação da questão & & & $x$ & $\mathrm{X}$ & & & & \\
\hline Referências bibliográficas & $\mathrm{X}$ & & & $\mathrm{X}$ & $\mathrm{x}$ & & & \\
\hline Regra/indicação de Prioridade & $x$ & & & $\mathrm{X}$ & $x$ & & & \\
\hline Regra/indicação de Aplicabilidade & $\mathrm{x}$ & & & & $x$ & & & \\
\hline Qualidade associada & $\mathrm{X}$ & $\mathrm{X}$ & $\mathrm{x}$ & $\mathrm{X}$ & $\mathrm{x}$ & & $\mathrm{X}$ & $\mathbf{X}$ \\
\hline Componente associado & $x$ & & & $\mathrm{X}$ & $x$ & & $\mathrm{X}$ & $\mathbf{x}$ \\
\hline \multicolumn{9}{|l|}{ Tipos de Respostas } \\
\hline SIM & $\mathrm{x}$ & $x$ & $x$ & $\mathrm{X}$ & $\mathrm{x}$ & & $\mathrm{x}$ & $\mathbf{x}$ \\
\hline NÃO & $\mathrm{X}$ & $\mathrm{x}$ & $x$ & $\mathrm{X}$ & $\mathrm{x}$ & & $\mathrm{X}$ & $\mathbf{x}$ \\
\hline Parcial & $\mathrm{x}$ & $\mathrm{x}$ & $x$ & $\mathrm{X}$ & & & & \\
\hline Não Aplicável & $\mathrm{x}$ & $\mathrm{x}$ & $\mathrm{x}$ & $\mathrm{X}$ & & & $\mathrm{X}$ & \\
\hline \multicolumn{9}{|l|}{ No opinion } \\
\hline Nat. Observável & & & & & $\mathrm{X}$ & & & \\
\hline Nat. Não Observável & & & & & $x$ & & & \\
\hline \multicolumn{9}{|l|}{ Nível de Aderência } \\
\hline Nível de Severidade & & & & & $\mathrm{X}$ & $\mathrm{x}$ & & \\
\hline Comentários & $\mathrm{X}$ & $\mathrm{X}$ & & $\mathrm{X}$ & $x$ & $\mathrm{x}$ & & \\
\hline Exemplos concretos de desconformidades & $\mathrm{X}$ & & & & & & & \\
\hline \multicolumn{9}{|l|}{ Material de apoio } \\
\hline Treinamento & $\mathrm{x}$ & & & & & & & \\
\hline Manual de aplicação & $\mathrm{X}$ & & $x$ & $\mathrm{X}$ & & & & \\
\hline Tabela resumo de aplicação & $x$ & & & & & & & \\
\hline Site $W e b$ & & $\mathrm{X}$ & & $\mathrm{X}$ & & & $\mathrm{X}$ & $\mathbf{x}$ \\
\hline Ferramenta de apoio & & & & & $X$ & $\mathrm{X}$ & $\mathrm{X}$ & $\mathbf{x}$ \\
\hline Padrões de documentos & & & & & $x$ & $x$ & & \\
\hline \multicolumn{9}{|l|}{ Procedimentos de Aplicação } \\
\hline Análise do contexto & $\mathrm{X}$ & $\mathrm{X}$ & $x$ & $\mathrm{X}$ & $\mathrm{X}$ & $\mathrm{X}$ & $\mathrm{X}$ & $\mathbf{x}$ \\
\hline Execução segundo casos de uso & $x$ & $\mathrm{x}$ & $x$ & $\mathrm{X}$ & $x$ & $x$ & $\mathrm{X}$ & $\mathbf{x}$ \\
\hline
\end{tabular}




\begin{tabular}{|c|c|c|c|c|c|c|c|c|}
\hline Execução segundo estrutura/componentes & $\mathrm{X}$ & $\mathrm{X}$ & & & $\mathrm{X}$ & $\mathrm{X}$ & $\mathrm{X}$ & $\mathbf{X}$ \\
\hline Execução do software pelo avaliador & $X$ & $X$ & $X$ & $\mathrm{X}$ & $X$ & $X$ & $X$ & $\mathbf{x}$ \\
\hline Execução do software pelo operador & $\mathrm{X}$ & $\mathrm{X}$ & & & $\mathrm{X}$ & $\mathrm{X}$ & $\mathrm{X}$ & $\mathbf{X}$ \\
\hline Aplicação posterior a execução do software & $\mathrm{X}$ & & $\mathrm{X}$ & & $\mathrm{X}$ & $\mathrm{X}$ & $\mathrm{X}$ & $\mathbf{x}$ \\
\hline Aplicação concorrente a execução do software & & $\mathrm{X}$ & & $\mathrm{X}$ & & & $x$ & $\mathbf{x}$ \\
\hline Aplicação posterior e concorrente & & $\mathrm{X}$ & & $\mathrm{X}$ & & & $\mathrm{X}$ & $\mathbf{X}$ \\
\hline \multicolumn{9}{|l|}{ Tratamento quantitativo } \\
\hline Definição de Importâncias relativas & & & $x$ & & $\mathrm{X}$ & $\mathrm{X}$ & & \\
\hline Definição de Aplicabilidade & $\mathrm{X}$ & & & $\mathrm{X}$ & $x$ & $\mathrm{x}$ & & $\mathbf{X}$ \\
\hline Definição de Aderência & $X$ & $\mathrm{X}$ & $\mathrm{X}$ & $x$ & $X$ & $X$ & $\mathrm{X}$ & $\mathbf{x}$ \\
\hline Reuniões de Integração de relatórios & & & & & & $\mathrm{X}$ & & \\
\hline Entrevistas de Integração de relatórios & & & & & & $X$ & & \\
\hline Medição do esforço da avaliação & & & & & & $X$ & & \\
\hline \multicolumn{9}{|l|}{ Relatórios } \\
\hline Técnico final do avaliador (interno) & & & & & & $\mathrm{X}$ & & \\
\hline Técnico final do grupo (interno) & & & & & & $X$ & & \\
\hline Laudo Final da Avaliação (externo) & & & & & & $x$ & $\mathrm{X}$ & \\
\hline Relatório gerencial (interno) & & & & & & $\mathbf{X}$ & & \\
\hline
\end{tabular}

Tabela 02: Estrutura padrão das listas de verificação. Fonte: (DYCK, 2005) adaptado pelos autores

Ao analisar os dados acima em conjunto com as heurísticas de usabilidade é perceptível a falta de uma lista de verificação de usabilidade específica para avaliar as interfaces de smartphones com foco nas necessidades do público idoso.

\section{Materiais e Métodos.}

A pesquisa foi desenvolvida em seis etapas iniciadas pela pesquisa bibliográfica para levantamento do estado da arte dos estudos sobre heurísticas de usabilidade. O passo seguinte foi a análise comparativa das heurísticas, assim como das listas de verificação encontradas durante a pesquisa bibliográfica. Após a comparação das publicações, foi desenvolvida uma lista de verificação preliminar a ser testada e validada por um grupo de especialistas. Em seguida, foram feitos ajustes na lista preliminar e disponibilizada publicamente para utilização.

\subsection{Pesquisa bibliográfica}

A revisão sistemática da literatura iniciou-se a partir da definição das áreas temáticas: design, usabilidade e envelhecimento de modo a identificar técnicas e métodos capazes de fornecer bases para avaliar questões relativas ao sistema e ao público, específicos dessa pesquisa.

Foram definidas as seguintes palavras-chave: "Elder", "older", "smartphone", "usability" e "heuristics" e foi perceptível que publicações com esse escopo tão fechado eram mais raras, portanto, foram suprimidos os termos "Elder" e "older" de modo a identificar o maior número de heurísticas de usabilidade disponíveis para tratar de smartphones para qualquer público.

As bases de dados utilizadas foram: SCOPUS, Periódicos CAPES e Google Scholar e o período de busca foi limitado a 2012 e 2019, por ser um período de grande popularização dessa categoria de artefato digital smartphone - e uma nova exploração entre 2020 e 2021 que trouxe dados sobre a inserção dos idosos na cultura digital, forçada pela pandemia da COVID-19. 
Foram identificadas publicações sobre heurísticas, princípios e diretrizes genéricos e específicos que buscavam atender a uma parcela de público no uso de determinados produtos, além disso, foi possível perceber que o número de publicações tem alterações no interesse de acordo com o ano pesquisado. Ao todo foram identificadas 61 publicações com as palavras-chave utilizadas e selecionadas 26 por tratarem de assuntos relacionados ao tema dessa pesquisa: idosos, Usabilidade e Smartphone de forma conjunta ou separadamente, mas com relevância para a pesquisa.

A figura a seguir traz os resultados da revisão da literatura classificados de acordo com o número de citações encontradas nas referências bibliográficas dos artigos, critério utilizado para seleção das heurísticas abordadas:

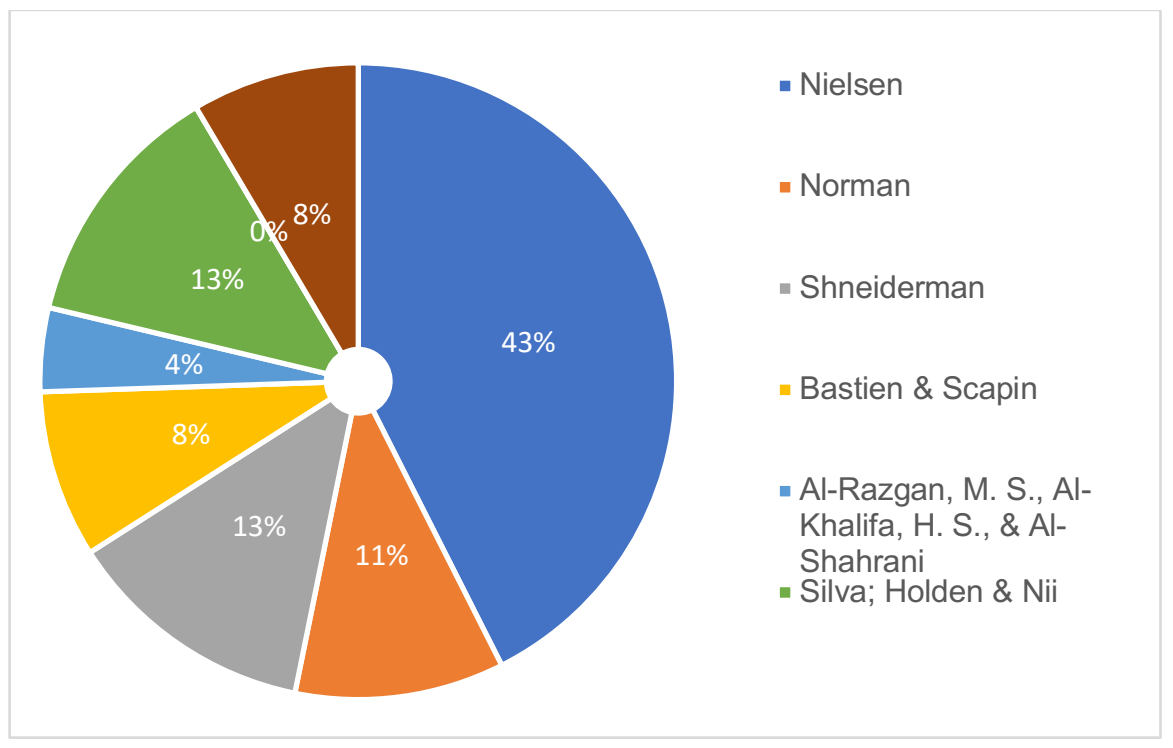

Figura 01: Gráfico de citações a heurísticas na Revisão Sistemática. Fonte: Os autores, 2019

A figura acima é um retrato do número de citações encontradas 'as heurísticas propostas pelos autores da coluna da direita. Os princípios de usabilidade de Nielsen são citados em 20 das 22 publicações, principalmente pela sua importância e pioneirismo, as outras citações se devem 'as suas especificidades e aplicabilidade adequada aos artefatos e ao público em questão. A tabela a seguir, além de listas as heurísticas citadas, as classifica como gerais ou específicas de acordo com sua aplicabilidade:

\begin{tabular}{|c|c|c|c|}
\hline HEURÍSTICA, PRINCÍPIO OU DIRETRIZ & AUTOR & $\begin{array}{l}\text { GERAL / DOMÍNIO } \\
\text { ESPECÍFICO }\end{array}$ & $\begin{array}{l}\text { QUANTIDADE } \\
\text { DE CITAÇÕES }\end{array}$ \\
\hline HEURÍSTICAS DE NIELSEN & Jakob Nielsen & Geral & 24 \\
\hline REGRAS DE OURO DE SHNEIDERMAN & Ben Shneiderman & Geral & 6 \\
\hline $\begin{array}{l}\text { HEURISTICS FOR SMARTPHONE } \\
\text { LAUNCHERS TARGETED AT OLDER } \\
\text { ADULTS. }\end{array}$ & Silva; Holden \& Nii & Específico & 7 \\
\hline PRINCÍPIOS DE NORMAN & Donald Norman & Geral & 5 \\
\hline $\begin{array}{l}\text { CRITÉRIOS ERGONÔMICOS DE } \\
\text { USABILIDADE }\end{array}$ & $\begin{array}{l}\text { Dominique Scapin e Christian } \\
\text { Bastien }\end{array}$ & Geral & 4 \\
\hline HEURÍSTICAS DE AVALIAÇÃO MÓVEL & Gomez et al & Específico & 4 \\
\hline $\begin{array}{l}\text { HEURISTICS FOR EVALUATING } \\
\text { THE USABILITY OF MOBILE LAUNCHERS } \\
\text { FOR ELDERLY PEOPLE. }\end{array}$ & $\begin{array}{l}\text { Al-Razgan, M. S., Al-Khalifa, H. } \\
\text { S., \& Al-Shahrani }\end{array}$ & Específico & 4 \\
\hline $\begin{array}{l}\text { RECOMENDAÇÕES DE USABILIDADE } \\
\text { PARA INTERFACE VISANDO O PÚBLICO } \\
\text { IDOSO. }\end{array}$ & Anjos \& Gontijo & Específico & 1 \\
\hline
\end{tabular}

Tabela 03: Lista de heurísticas referenciadas na revisão da literatura. Fonte: os autores, 2019 
É possível identificar as referências recorrentes às primeiras heurísticas, independentemente da especificidade que se propõe com a nova o que pode sinalizar que, por mais que se criem possibilidades, a base será sempre importante como ponto de partida e, também, sinaliza a não obsolescência da proposta inicial.

\subsection{Criação de lista de verificação}

Após a análise comparativa de heurísticas e a análise comparativa de listas de verificação, foi possível identificas itens comuns, que atendem às necessidades específicas do público idoso, assim como a ausência de recomendações de usabilidade destinadas ao público.

As diretrizes foram divididas em quatro grupos: facilidade de leitura; interação; memória; e funcionalidades. Essas categorias são baseadas nas principais dificuldades encontradas pelos idosos no uso dos smartphones de acordo com (AL-RAZGAN, AL-KHALIFA, \& AL-SHAHRANI, 2014).

Essa lista de verificação foi validada posteriormente por um grupo de especialistas em design e usabilidade a partir da criação de um website disponível para utilização gratuita por designers e desenvolvedores que necessitem averiguar se seus sistemas e aplicativos possuem boa usabilidade para idosos. Para isso, foi criado um sistema de pontuação que, de acordo com as respostas coletadas, apresentará uma nota.

Cada questão possui um número de pontos específico para serem somados ao total, no final das respostas. As respostas Sim, valem 3 pontos, as respostas Parcialmente, valem 2 pontos, já as respostas Não Aplicável, valem 1 ponto e a resposta Não, vale 0 pontos. Essa pontuação total vai de 0 a 129, na qual 0 é Inadequado, para o uso de idosos e 129 caracteriza um sistema Adequado para o uso do público em questão. A lista completa, com as sugestões dos especialistas será explicitada a seguir.

\section{Resultados}

Durante a pesquisa bibliográfica foram identificadas diversas listas de verificação de usabilidade, mas nenhuma delas contemplava a usabilidade de smartphones para o público idoso. Apenas na lista ACESSIBILIDADE, havia uma preocupação maior com o público idoso, mas essa lista havia sido desenvolvida para avaliação de usabilidade em computadores de mesa.

O desenvolvimento da LUSUI se baseou nas necessidades específicas do público e na objetividade da análise específica do público idoso. Por haver um número grande de listas e heurísticas de usabilidade, pode não haver um direcionamento mais específico da análise para pontos específicos, portanto, justificou-se a criação da ferramenta descrita nessa pesquisa.

Após a validação dos especialistas, a lista passou por algumas modificações como a redução de questões redundantes e melhoria na elaboração delas. Ao final, ela fica composta de 43 questões divididas em quatro categorias: Facilidade de Leitura, Interação, Memória e Funcionalidades.

\subsection{Validação da lista de verificação}

A Lista de Verificação foi enviada para sete especialistas em usabilidade para validação. Foi solicitado a eles que fizessem a avaliação de um sistema móvel utilizando a lista proposta. Ao final de cada uma das quatro categorias, eles poderiam descrever suas impressões e fazer observações sobre a estrutura das questões e a viabilidade delas. Além disso, no final do questionário, além da nota atribuída ao sistema, será possível inserir mais informações, sugestões e críticas sobre todo o procedimento adotado e a metodologia do teste. 
A lista foi validada com sete especialistas: um Designer com especialização em ergodesign para interfaces digitais; um consultor de segurança da informação; um UX designer; uma Professora Universitária de Design de Interação; um mestre em design de artefatos digitais; e dois designers com experiência em UI Design.

\subsection{Versão Final da LUSUI}

Após a análise e validação por especialistas, foram feitos alterações e agrupamentos nas categorias para reduzir quantidade de questões e tornar a lista mais objetiva

Facilidade de Leitura: Trata de questões relativas ao sistema sensorial visual e à visibilidade e legibilidade dos elementos da interface (botões, texto, atalhos, links).

A1. Os elementos possuem bom contraste entre eles e o fundo?

\begin{tabular}{|lll|}
\hline ( ) Sim ( ) Parcialmente & ( ) Não ( ) Não aplicável \\
\hline Observações: & \\
\hline
\end{tabular}

A2. O sistema possibilita a configuração de acessibilidade e adaptabilidade às necessidades especiais dos usuários?

\begin{tabular}{|lll|}
\hline ( ) Sim & ( ) Parcialmente $\quad$ ( ) Não ( ) Não aplicável \\
\hline Observações: & \\
\hline
\end{tabular}

A3. Os botões e imagens clicáveis possuem tamanho que facilita a interação do idoso?

\begin{tabular}{|lll|}
\hline ( ) Sim & ( ) Parcialmente & ( ) Não ( ) Não aplicável \\
\hline Observações: & \\
\hline
\end{tabular}

A4. Os ícones possuem formas que remetem a objetos e ações que fazem parte do repertório dos idosos?

\begin{tabular}{|lll|}
\hline ( ) Sim & ( ) Parcialmente & ( ) Não ( ) Não aplicável \\
\hline Observações: & \\
\hline
\end{tabular}

A5. Os textos possuem tamanho mínimo de 14 pontos?

\begin{tabular}{|lll|}
\hline ( ) Sim & ( ) Parcialmente & ( ) Não ( ) Não aplicável \\
\hline Observações: & \\
\hline
\end{tabular}

A6. A linguagem das ações e áreas do sistema possuem linguagem simplificada?

\begin{tabular}{|lll|}
\hline ( ) Sim ( ) Parcialmente $\quad$ ( ) Não ( ) Não aplicável \\
\hline Observações: & \\
\hline
\end{tabular}

A7. O número elementos visuais na interface é reduzido?

\begin{tabular}{|lll|}
\hline ( ) Sim ( ) Parcialmente & ( ) Não ( ) Não aplicável \\
\hline Observações: & \\
\hline
\end{tabular}

A8. O sistema é capaz de ser configurado e se adaptar à forma de utilização?

\begin{tabular}{|lll|}
\hline ( ) Sim Sim ( ) Parcialmente & ( ) Não ( ) Não aplicável \\
\hline Observações: & \\
\hline
\end{tabular}

A9. O sistema oferece leitor de tela por voz?

\begin{tabular}{|lll|}
\hline ( ) Sim & ( ) Parcialmente & ( ) Não ( ) Não aplicável \\
\hline Observações: & \\
\hline
\end{tabular}


A10. O sistema evita utilizar as cores como única diferenciação entre categorias?

\begin{tabular}{|lll|}
\hline ( ) Sim ( ) Parcialmente & ( ) Não ( ) Não aplicável \\
\hline Observações: & \\
\hline
\end{tabular}

A11. O sistema oferece facilmente a opção de configurações de acessibilidade?

\begin{tabular}{|lll|}
\hline ( ) Sim ( ) Parcialmente & ( ) Não ( ) Não aplicável \\
\hline Observações: & \\
\hline
\end{tabular}

A12. Os itens possuem espaçamento suficiente entre eles para garantir a boa leitura?

\begin{tabular}{|lll|}
\hline ( ) Sim ( ) Parcialmente & ( ) Não ( ) Não aplicável \\
\hline Observações: & \\
\hline
\end{tabular}

A13. Há redução do número de animações e movimentos rápidos?

\begin{tabular}{|lll|}
\hline ( ) Sim ( ) Parcialmente & ( ) Não ( ) Não aplicável \\
\hline Observações: & \\
\hline
\end{tabular}

Interação: Trata da forma como o usuário navega pelo sistema, formas de tocar, movimentos de dedos para determinadas ações, respostas do sistema aos comados.

B1. O sistema responde corretamente aos comandos do usuário?

\begin{tabular}{|lll|}
\hline ( ) Sim ( ) Parcialmente & ( ) Não ( ) Não aplicável \\
\hline Observações: & \\
\hline
\end{tabular}

B2. O sistema possui regulagem de sensibilidade ao toque?

\begin{tabular}{|lll|}
\hline ( ) Sim ( ) Parcialmente & ( ) Não ( ) Não aplicável \\
\hline Observações: & \\
\hline
\end{tabular}

B3. Todas as ações emitem feedback sonoro, tátil e visual?

\begin{tabular}{|lll|}
\hline ( ) Sim ( ) Parcialmente & ( ) Não ( ) Não aplicável \\
\hline Observações: & \\
\hline
\end{tabular}

B4. O sistema evita o uso de movimentos complexos como pinçar e arrastar para tarefas mais básicas?

\begin{tabular}{|lll|}
\hline ( ) Sim ( ) Parcialmente $\quad$ ( ) Não ( ) Não aplicável \\
\hline Observações: & \\
\hline
\end{tabular}

B5. O Teclado do sistema oferece palavras comumente utilizadas em botões de tamanho maior?

\begin{tabular}{|lll|}
\hline ( ) Sim ( ) Parcialmente & ( ) Não ( ) Não aplicável \\
\hline Observações: & \\
\hline
\end{tabular}

B6. O sistema permite o fácil compartilhamento de fotografias?

\begin{tabular}{|lll|}
\hline ( ) Sim & ( ) Parcialmente & ( ) Não ( ) Não aplicável \\
\hline Observações: & \\
\hline
\end{tabular}

B7. Os sons utilizados possuem frequências mais altas e melodias simples?

\begin{tabular}{|lll|}
\hline ( ) Sim ( ) Parcialmente & ( ) Não ( ) Não aplicável \\
\hline Observações: & \\
\hline
\end{tabular}


B8. O sistema oferece informações objetivas sobre as funções que irá executar (fotografar, enviar e-mail, telefonar, etc.)?

\begin{tabular}{|lll|}
\hline ( ) Sim ( ) Parcialmente & ( ) Não ( ) Não aplicável \\
\hline Observações: & \\
\hline
\end{tabular}

B9. Todas as telas mantêm o padrão visual?

\begin{tabular}{|lll|}
\hline ( ) Sim ( ) Parcialmente & ( ) Não ( ) Não aplicável \\
\hline Observações: & \\
\hline
\end{tabular}

B10. O sistema considera o posicionamento natural dos dedos durante o manuseio para os ícones?

\begin{tabular}{|lll|}
\hline ( ) Sim & ( ) Parcialmente & ( ) Não ( ) Não aplicável \\
\hline Observações: & \\
\hline
\end{tabular}

B11. O sistema informa ao usuário em que lugar ele está (configuração, câmera, mensagens, etc.)?

\begin{tabular}{|lll|}
\hline ( ) Sim & ( ) Parcialmente & ( ) Não ( ) Não aplicável \\
\hline Observações: & \\
\hline
\end{tabular}

B12. O sistema possibilita o uso de diferentes formas de notificação e feedback? (vibração, sons, imagens, flash)?

\begin{tabular}{|lll|}
\hline ( ) Sim $\quad$ ( ) Parcialmente $\quad$ ( ) Não ( ) Não aplicável \\
\hline Observações: & \\
\hline
\end{tabular}

B13. O sistema evita o uso de menus suspensos?

\begin{tabular}{|lll|}
\hline ( ) Sim ( ) Parcialmente $\quad$ ( ) Não ( ) Não aplicável \\
\hline Observações: & \\
\hline
\end{tabular}

B14. O sistema permite que todas as informações estejam disponíveis em uma tela apenas, sem a necessidade de rolar para ver mais?

\begin{tabular}{|lll|}
\hline ( ) Sim $\quad$ ( ) Parcialmente & ( ) Não ( ) Não aplicável \\
\hline Observações: & \\
\hline
\end{tabular}

Memória: com o avanço da idade é perceptível que a memória é afetada, para isso é necessário que os sistemas sejam pensados de forma que não exijam tanto esforço do usuário idoso em lembrar de detalhes.

C1. O sistema informa ao usuário em que ponto da atividade ele parou?

\begin{tabular}{|lll|}
\hline ( ) Sim ( ) Parcialmente & ( ) Não ( ) Não aplicável \\
\hline Observações: & \\
\hline
\end{tabular}

C2. O sistema possibilita a gravação do estado da atividade para recuperação caso haja interrupção?

\begin{tabular}{|lll|}
\hline ( ) Sim ( ) Parcialmente & ( ) Não ( ) Não aplicável \\
\hline Observações: & \\
\hline
\end{tabular}

C3. Permite que o usuário desfaça ações executadas sem querer ou equivocadas?

\begin{tabular}{|lll|}
\hline ( ) Sim ( ) Parcialmente & ( ) Não ( ) Não aplicável \\
\hline Observações: & \\
\hline
\end{tabular}

C4. As funções que executam tarefas semelhantes como fotografar e editar foto, navegar na internet e enviar e-mail são agrupadas na tela?

\begin{tabular}{|ll|}
\hline ( ) Sim ( ) Parcialmente $\quad$ ( ) Não ( ) Não aplicável \\
\hline Observações: & \\
\hline
\end{tabular}


C5. As funções básicas ficam disponíveis facilmente para o usuário?

\begin{tabular}{|lll|}
\hline ( ) Sim & ( ) Parcialmente & ( ) Não ( ) Não aplicável \\
\hline Observações: & \\
\hline
\end{tabular}

C6. O sistema considera o conhecimento do usuário nas suas ações?

\begin{tabular}{|lll|}
\hline ( ) Sim ( ) Parcialmente & ( ) Não ( ) Não aplicável \\
\hline Observações: & \\
\hline
\end{tabular}

C7. O sistema limita as ações a uma por vez?

\begin{tabular}{|ll|}
\hline ( ) Sim ( ) Parcialmente & ( ) Não ( ) Não aplicável \\
\hline Observações: & \\
\hline
\end{tabular}

Funcionalidades: O sistema precisa oferecer resoluções para situações causadas por ele ou pelo usuário, prevenir erros, reconhecer comandos e oferecer ajuda ao usuário em qualquer etapa de utilização.

D1. O sistema permite o reconhecimento de comandos por voz?

\begin{tabular}{|lll|}
\hline ( ) Sim & ( ) Parcialmente & ( ) Não ( ) Não aplicável \\
\hline Observações: & \\
\hline
\end{tabular}

D2. O sistema permite a configuração de atalhos de emergência?

\begin{tabular}{|lll|}
\hline ( ) Sim & ( ) Parcialmente & ( ) Não ( ) Não aplicável \\
\hline Observações: & \\
\hline
\end{tabular}

D3. O sistema informa e confirma antes da execução de ações para evitar que elas não possam ser desfeitas?

\begin{tabular}{|lll|}
\hline ( ) Sim & ( ) Parcialmente & ( ) Não ( ) Não aplicável \\
\hline Observações: & \\
\hline
\end{tabular}

D4. O sistema apresenta informações consistentes de resolução de erros?
( ) $\operatorname{Sim}$
( ) Parcialmente
( ) Não ( ) Não aplicável
Observações:

D5. O sistema informa o estado de funções como wi-fi, sinal de celular, Bluetooth, estado da bateria?
( ) $\operatorname{Sim}$
( ) Parcialmente
Observações:
( ) Não ( ) Não aplicável

D6. O sistema oferece possibilidades de obter ajuda durante a utilização em qualquer tela?

\begin{tabular}{|lll|}
\hline ( ) Sim ( ) Parcialmente & ( ) Não ( ) Não aplicável \\
\hline Observações: & \\
\hline
\end{tabular}

D7. O sistema oferece mais informações sobre suas funções?

\begin{tabular}{|lll|}
\hline ( ) Sim ( ) Parcialmente & ( ) Não ( ) Não aplicável \\
\hline Observações: & \\
\hline
\end{tabular}

D8. O usuário sente que está no controle do sistema?

\begin{tabular}{|lll|}
\hline ( ) Sim $\quad$ ( ) Parcialmente & ( ) Não ( ) Não aplicável \\
\hline Observações: & \\
\hline
\end{tabular}

D9. O sistema oferece boa proteção de dados e deixa isso claro para o usuário?

\begin{tabular}{|lll|}
\hline ( ) Sim ( ) Parcialmente & ( ) Não ( ) Não aplicável \\
\hline Observações: & \\
\hline
\end{tabular}




\subsection{Discussão dos resultados}

Ferramentas de apoio ao projeto de interfaces podem trazer uma maior facilidade para os desenvolvedores. Uma lista de verificação de usabilidade pode ser aplicada em uma etapa inicial de desenvolvimento de aplicativos ou sistemas operacionais e guiar os designers em um percurso mais objetivo, com menos correções e redução dos possíveis erros.

Foi produzida uma imagem (Figura 02) que traz a rede de conexões entre as listas de verificação - de cor bordô - e as heurísticas de usabilidade - na cor azul - estudadas para ilustrar a interdependência entre as duas ferramentas. Durante a pesquisa bibliográfica, foram feitas conexões entre as diretrizes e recomendações propostas pelas heurísticas avaliadas - representadas pela cor bege - que traz à tona a consonância do pensamento dos seus criadores, que se preocupam com questões semelhantes, mesmo não sendo tão específicas para smartphones ou idosos.

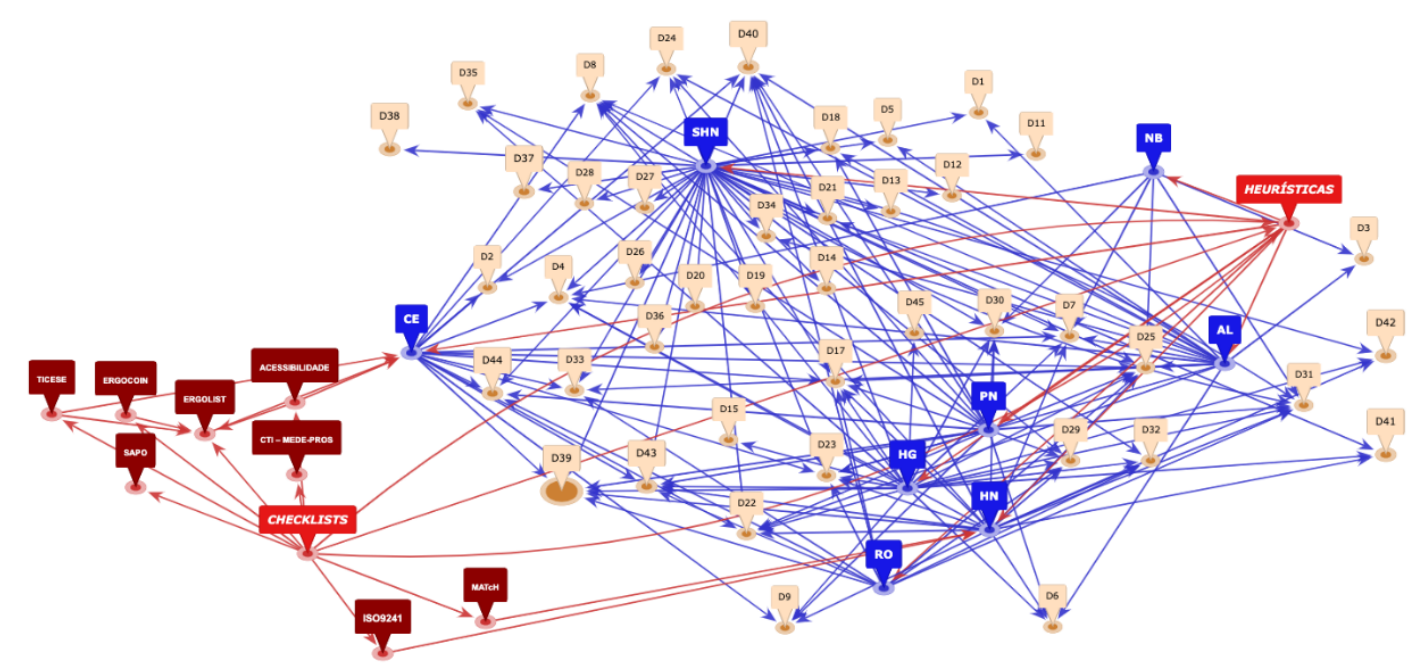

Figura 02: Rede de Conexões entre Heurísticas e Listas de Verificação (OS AUTORES,2021)

Já sobre as listas de verificação, foi possível observar que elas foram geradas a partir das principais heurísticas estudadas e que sintetizam as diretrizes trazidas por ela, as três desenvolvidas pelo LabIUtil são derivadas diretamente dos Critérios Ergonômicos de Usabilidade - CE, já a MATcH e a ISO9241 têm requisitos muito semelhantes às heurísticas de Nielsen - HN.

A LUSUI se propõe a ser esse guia de desenvolvimento nas fases iniciais para o desenho de interfaces não somente para os idosos, mas que contemple esse público de forma satisfatória já nas fases iniciais de desenvolvimento evitando correções em etapas futuras. Como pode ser utilizada na fase inicial, ainda antes do desenvolvimento dos primeiros protótipos e da versão funcional da interface, há uma redução de custos para a equipe.

\section{Considerações Finais}

Por mais que o número de idosos tenha sofrido uma diminuição em virtude da pandemia do novo coronavírus, por conta da qualidade de vida e da saúde das pessoas, a população irá envelhecer cada vez mais e pensar soluções para os idosos é um tema atual e carece de atenção. 
Sabendo que esse público nem sempre é acessível para pesquisas de usabilidade e para facilitar o desenvolvimento de novos aplicativos e sistemas, a criação de uma lista de verificação de usabilidade pode auxiliar na melhoria da qualidade e na usabilidade dos artefatos para eles.

Foi criada uma lista de verificação online que contempla quatro categorias de avaliação - Facilidade de Leitura, Interação, Memória e Funcionalidades - divididas em 45 questões que pretendem facilitar o desenvolvimento de interfaces de smartphones com melhor acessibilidade para idosos, sem reduzir a acessibilidade de outros usuários.

A lista foi validada por um grupo de especialistas em design, usabilidade e tecnologia e foram feitas adaptações de acordo com as observações desses especialistas, resultando em 43 questões - foram excluídas duas questões consideradas redundantes pelos especialistas - que seguem contemplando as quatro áreas de avaliação propostas anteriormente. A pesquisa busca propor a lista como uma ferramenta adicional para facilitar o projeto de interfaces visando atender os idosos.

O sistema de pontuação criado para essa avaliação pode criar um ranking que pode, inclusive, ser divulgado como referência no lançamento de produtos voltados para esse público. Ainda assim, recomenda-se que sejam realizados estudos mais voltados para a validação dessas notas com base em outros sistemas de mensuração de usabilidade.

Durante o desenvolvimento da pesquisa foi possível identificar alguns estudos sobre a usabilidade de sistemas com enfoque nos idosos, mas ainda assim são necessários novos trabalhos voltados a esse público e sua inserção na cultura digital, buscando trazer à luz, soluções para problemas como isolamento, solidão e depressão que muitas vezes acomete os idosos.

Smartphones possuem, em sua maioria, configurações voltadas para a acessibilidade, mas essas opções nem sempre são claras ou fáceis de encontrar por quem não tem domínio de tecnologias, isso sinaliza a necessidade de criar formas de interação que direcionem o idoso para a configuração correta assim que iniciar o smartphone pela primeira vez, evitando a necessidade de auxílio de outras pessoas.

As listas de verificação se propõem a ser uma ferramenta de aplicação prática das heurísticas, evitando a subjetividade dos avaliadores. A LUSUI se coloca como ferramenta de apoio ao desenvolvimento de aplicativos e sistemas com foco no idoso já nas fases iniciais, para evitar problemas comuns a esse público. Além disso, por conta de sua replicabilidade no meio digital, a LUSUI possibilita a sua aplicação em diferentes contextos sociais e culturais em qualquer lugar do mundo, a partir de sua tradução. O que não significa que testes e avaliações com o público sejam descartados pois, são essenciais para uma boa usabilidade de um sistema. Assim, a Lista de Verificação possui limitações por não inserir o usuário em sua execução.

É importante ressaltar que existem perfis diferenciados de idosos que, de acordo com o avançar da idade têm maiores dificuldades na execução de tarefas por conta do aumento das deficiências já iniciadas por volta dos 60 anos. Os super idosos, como são conhecidos, podem trazer novos dados em pesquisas futuras mais focadas na maior degeneração das capacidades trazida com o avanço da idade.

Além disso, cabe ressaltar que parte da experiência do usuário vem do artefato físico smartphone e que o idos tem questões próprias relativas à dificuldade do sistema háptico, que pode influenciar no seu uso, principalmente em uma evolução gradativa de smartphones que traz modelos cada vez maiores e mais finos, que podem dificultar o manejo do aparelho. Essa questão não foi abordada nesse estudo e carece de análises ergonômicas focadas no uso do aparelho em si em consonância com a interface na execução de tarefas.

Como desdobramentos dessa pesquisa, serão investigadas questões metodológicas da área do design e da Interação Humano-Computador para compreender melhor como são criadas as tecnologias voltadas para o 
bem-estar do idoso, conhecidas como Gerontecnologia, que abrange áreas multidisciplinares preocupadas com a temática.

\section{Referências}

ABNT. (2011). NBR ISO 9241. Rio de Janeiro: ABNT.

AGING IN PLACE. (2021). Cellphone Guide for Seniors. Fonte: Aging in Place:

https://aginginplace.org/cellphone-guide-for-seniors/

AL-RAZGAN, M., AL-KHALIFA, H., \& AL-SHAHRANI, M. (2014). Heuristics for evaluating the usability of mobile launchers for elderly people. Theories, Methods, and Tools for designing the user experience Lecture Notes in Computer Science, 8517, 415-424.

ANJOS, T. P., \& GONTIJO, L. A. (2015). Recomendações de usabilidade e acessibilidade para interface de telefone celular visando o público idoso. Production, 25(4), 20.

BASTIEN, J., \& SCAPIN, D. (1993). Ergonomic Criteria for the Evaluation of Human-Computer interfaces. Institut National de recherche en informatique et en automatique.

BO XIE, NEIL CHARNESS, KAREN FINGERMAN, JEFFREY KAYE, MIYONG T. KIM \& ANJUM KHURSHID (2020) When Going Digital Becomes a Necessity: Ensuring Older Adults' Needs for Information, Services, and Social Inclusion During COVID-19, Journal of Aging \& Social Policy, 32:4-5, 460-470, DOI: 10.1080/08959420.2020.1771237

BRYKCZYNSKI, B. (1999). A Survey of Software Inspection Checklists. Software Engineering Notes, 24(1), pp. 82-89.

CIBYS, W., BETIOL, A., \& FAUST, R. (2017). ERGONOMIA E USABILIDADE: Conceitos, Métodos e Aplicações (3a Edição ed.). São Paulo: Novatec Editora.

CIBYS, W. A., DYCK, A. F., OLIVEIRA, R., PAGLIUSO, P. B., TAMBASCIA, C. A., \& VILLAS-BOAS, A. L. (2003). Approach for the development of systematic and productive verifying lists of usability. Proceedings of the Latin American conference on Human-computer interaction (pp. 29-40). Nova Iorque: ACM.

CNN. (2020) Quarentena acelera inclusão digital de idosos. Acessado em 31/07/2021. Disponível em: $<$ https://www.cnnbrasil.com.br/nacional/2020/05/14/quarentena-acelera-inclusao-digital-de-idosos>

DYCK, A. F. (2005). Proposta de abordagem para o desenvolvimento de listas de verificação de usabilidade sistemáticas e produtivas, 256f. Florianópolis, SC, Brasil: UFSC.

GÓMEZ, R. Y., CABALLERO, D. C., \& SEVILLANO, J. (2014). Heuristic Evaluation on Mobile Interfaces: A New Checklist. The Scientific World Journal, 2014, 19.

GAMEZ, L. (1998). TICESE - Técnica de Inspeção de conformidade Ergonômica de Software Educacional. Guimarães, Portugal: Universidade do Minho.

GQS-UFSC. (s.d.). Checklist para Avaliação da Usabilidade de Aplicativos para Celulares Touchscreen. Fonte: http://match.inf.ufsc.br:90/.

HOM, J. (2003). The Usability Toolbox. Acesso em 06 de 05 de 2019, disponível em jameshom.com: http://usability.jameshom.com/

IBGE. (2018). PNAD Contínua TIC 2017: Internet chega a três em cada quatro domicílios do país. Acesso em maio de 2020, disponível em AGÊNCIA DE NOTÍCIAS IBGE:

https://agenciadenoticias.ibge.gov.br/agencia-sala-de-imprensa/2013-agencia-de-noticias/releases/23445-pnadcontinua-tic-2017-internet-chega-a-tres-em-cada-quatro-domicilios-do-pais

ISO. (2010). Human-centered design for interactive systems (9241-210). 
LABIUTIL. (2002). Checklist para Avaliação de Acessibilidade da Web para Usuários Idosos. Fonte: http://www.labiutil.inf.ufsc.br/acessibilidade/index.htm

LABIUTIL. (2011). LabIUtil - Laboratório de Utilizabilidade. Fonte: ErgoList: http://www.labiutil.inf.ufsc.br/ergolist/

NIELSEN, J. (1994). Heuristic evaluation. Em Usability Inspection Methods. New York: John Wiley \& Sons. NIELSEN, J., \& BUDIU, R. (2014). Usabilidade Móvel. Rio de Janeiro: Elsevier.

NIELSEN, J., \& MOLICH, R. (1990). Heurístic evaluation of user interfaces. Proceedings of ACM IHC'90 Conference, (pp. 249-256). Seattle.

NORMAN, D. (2018). Design Emocional ( $1^{\circ}$ ed.). Rio de Janeiro: Rocco.

OPAS. (2018). Folha informativa - Envelhecimento e saúde . Acesso em 20 de maio de 2020, disponível em https://www.paho.org/bra/index.php?option=com_content\&view=article\&id=5661:folha-informativaenvelhecimento-e-saude $\&$ Itemid $=820$

PEW RESEARCH CENTER. (2017). PEW RESEARCH CENTER. Fonte: Tech Adoption Climbs Among Older Adults: https://www.pewresearch.org/internet/2017/05/17/tech-adoption-climbs-among-older-adults/

PEW RESEARCH CENTER. (2019). PEW RESEARCH CENTER. Fonte: Mobile Technology and Home Broadband 2019: https://www.pewresearch.org/internet/2019/06/13/mobile-technology-and-home-broadband2019/

PREECE, J., ROGERS, Y., \& SHARP, H. (2019). Interaction Design: Beyond human-computer interaction ( $5^{\circ}$ Edição ed.). Indianapolis: Wiley.

SHNEIDERMAN, B., \& PLAISANT, C. (2004). Designing the User Interface: Strategies for Effective Human-Computer Interaction ( $4^{\circ}$ Edição ed.). Pearson Addison Wesley.

SILVA, P. A., HOLDEN, K., \& NII, A. (2014). Smartphones, Smart Senior, but not-so-smart apps: A Heuristic Evaluation of fitness apps. D. D. Schmorrow, \& C. M. Fidopiastis (Eds.), Foundations of augmented cognition. Advancing human performance and decision-making through adaptive systems, 347358.

\section{Agradecimentos}

Ao Proidoso pela contribuição e disponibilidade em colaborar com a pesquisa e a todos os participantes que se dispuseram a responder todas as perguntas e se deixaram ser observados. 